\title{
A cohort of 17 patients with kyphoscoliotic Ehlers-Danlos syndrome caused by biallelic mutations in FKBP14: expansion of the clinical and mutational spectrum and description of the natural history
}

\author{
Cecilia Giunta, $\mathrm{PhD}^{1,23}$, Matthias Baumann, MD², Christine Fauth, MD³ , Uschi Lindert, $\mathrm{PhD}^{1}$, \\ Ebtesam M. Abdalla, MD, PhD ${ }^{4}$, Angela F. Brady, MD, PhD ${ }^{5}$, James Collins, MD, PhD ${ }^{6}$, \\ Jahannaz Dastgir, DO ${ }^{7}$, Sandra Donkervoort, MS, CGC ${ }^{8}$, Neeti Ghali, MD ${ }^{5}$, \\ Diana S. Johnson, BMBSCMD ${ }^{9}$, Ariana Kariminejad, MD ${ }^{10}$, Johannes Koch, MD ${ }^{11}$,
} Marius Kraenzlin, MD ${ }^{12}$, Nayana Lahiri, MD ${ }^{13}$, Bernarda Lozic, MD, PhD ${ }^{14}$, Adnan Y. Manzur, MD ${ }^{15}$, Jenny E.V. Morton, MD ${ }^{16}$, Jacek Pilch, MD, PhD ${ }^{17}$, Rebecca C. Pollitt, MSc ${ }^{18}$, Gudrun Schreiber, MD ${ }^{19}$, Nora L. Shannon, $\mathrm{MD}^{20}$, Glenda Sobey, $\mathrm{MD}^{9}$, Anthony Vandersteen, MD, $\mathrm{PhD}^{21}$,

Fleur S. van Dijk, MD, PhD ${ }^{5}$, Martina Witsch-Baumgartner, $\mathrm{PhD}^{3}$, Johannes Zschocke, $\mathrm{MD}, \mathrm{PhD}^{3}$,

F. Michael Pope, MD ${ }^{22}$, Carsten G. Bönnemann, $\mathrm{MD}^{8}$ and Marianne Rohrbach, MD, $\mathrm{PhD}^{1}$

Purpose: In 2012 we reported in six individuals a clinical condition almost indistinguishable from PLOD1-kyphoscoliotic EhlersDanlos syndrome (PLOD1-kEDS), caused by biallelic mutations in FKBP14, and characterized by progressive kyphoscoliosis, myopathy, and hearing loss in addition to connective tissue abnormalities such as joint hypermobility and hyperelastic skin. FKBP14 is an ER-resident protein belonging to the family of FK506-binding peptidyl-prolyl cis-trans isomerases (PPIases); it catalyzes the folding of type III collagen and interacts with type III, type VI, and type X collagens. Only nine affected individuals have been reported to date.

Methods: We report on a cohort of 17 individuals with FKBP14kEDS and the follow-up of three previously reported patients, and provide an extensive overview of the disorder and its natural history based on clinical, biochemical, and molecular genetics data.
Results: Based on the frequency of the clinical features of 23 patients from the present and previous cohorts, we define major and minor features of FKBP14-kEDS. We show that myopathy is confirmed by histology and muscle imaging only in some patients, and that hearing impairment is predominantly sensorineural and may not be present in all individuals.

Conclusion: Our data further support the extensive clinical overlap with PLOD1-kEDS and show that vascular complications are rare manifestations of FKBP14-kEDS.

Genet Med advance online publication 15 June 2017

Key Words: diagnostic criteria; FKBP14; FKBP22; kyphoscoliotic Ehlers-Danlos syndrome; mutations

\section{INTRODUCTION}

Ehlers-Danlos syndrome (EDS) comprises a heterogeneous spectrum of monogenic conditions with multisystemic and variable clinical manifestations affecting primarily the skin, ligaments and joints, blood vessels, and internal organs. Symptoms are usually present in early childhood and the clinical spectrum varies from mild skin and joint hyperlaxity to intermediate and severe physical disability and life-threatening complications. ${ }^{1,2}$

To date, the 1997 Villefranche classification of EDS has recognized six types based on clinical phenotype, inheritance

${ }^{1}$ Connective Tissue Unit, Division of Metabolism and Children's Research Centre, University Children's Hospital, Zurich, Switzerland; ${ }^{2}$ Department of Pediatrics I, Pediatric Neurology, Medical University of Innsbruck, Innsbruck, Austria; ${ }^{3}$ Division of Human Genetics, Medical University of Innsbruck, Innsbruck, Austria; ${ }^{4}$ Human Genetics Department, Medical Research Institute, Alexandria University, Alexandria, Egypt; ${ }^{5}$ Ehlers-Danlos Syndrome, National Diagnostic Service, Northwick Park and St. Mark's Hospitals, Harrow, UK; ${ }^{6}$ Mercy Clinic Pediatric Neurology, Springfield, Missouri, and Cincinnati Children's Hospital Medical Center, Cincinnati, Ohio, USA; ${ }^{7}$ Pediatric Neurology, Goryeb Children's Hospital, Morristown, New Jersey, USA; ${ }^{8}$ Neuromuscular and Neurogenetic Disorders of Childhood Section, National Institute of Neurological Disorders and Stroke, National Institutes of Health, Bethesda, Maryland, USA; ${ }^{9}$ Ehlers Danlos Syndrome National Diagnostic Service, Sheffield Children's Hospital, Sheffield, UK; ${ }^{10}$ Kariminejad-Najmabadi Pathology \& Genetics Center, Tehran, Iran; ${ }^{11}$ Department of Pediatrics, Paracelsus Medical University Salzburg, Salzburg, Austria; ${ }^{12}$ Medical Faculty of the University of Basel, and Clinic for Endocrinology, Diabetes \& Metabolism, University Hospital Basel, Basel, Switzerland; ${ }^{13}$ South West Thames Regional Genetics Service, St. George's University Hospitals NHS Foundation Trust, UK; ${ }^{14}$ Department of Pediatrics University Hospital Centre Split, Split, Croatia; ${ }^{15}$ Dubowitz Neuromuscular Centre, UCL Institute of Child Health, Great Ormond Street Hospital, London, UK; ${ }^{16}$ West Midlands Regional Clinical Genetics Service and Birmingham Health Partners Birmingham Women's Hospital NHS Foundation Trust, Birmingham, UK; ${ }^{17}$ Department of Pediatric Neurology, Medical University of Silesia, Katowice, Poland; ${ }^{18}$ Sheffield Diagnostic Genetics Service, Sheffield Children's NHS Foundation Trust, Sheffield, UK; ${ }^{19}$ Department of Pediatric Neurology, Children's Hospital, Kassel, Germany; ${ }^{20}$ Nottingham Clinical Genetics Service, Nottingham City Hospital, Nottingham, UK; ${ }^{21}$ Maritime Medical Genetics Service, IWK Health Centre, Halifax, Nova Scotia, Canada; ${ }^{22}$ North West Thames Regional Genetics Service, Kennedy Galton Centre, London, UK. Correspondence: Cecilia Giunta (Cecilia.Giunta@kispi.uzh.ch)

${ }^{23}$ The first two authors contributed equally to this work. 
pattern, and underlying biochemical and molecular defect(s). ${ }^{1}$ Among them, the kyphoscoliotic type of EDS (MIM 225400) was recognized as one distinct type based on the following characteristic clinical features: severe muscular hypotonia at birth; generalized joint laxity; kyphoscoliosis at birth, which is progressive; scleral fragility; and rupture of the ocular globe. The disorder, which is currently called by the 2017 International Nosology of EDS PLOD1-kyphoscoliotic EDS (PLOD1-kEDS), ${ }^{3}$ is caused by the deficiency of the collagen-modifying enzyme procollagen-lysine, 2-oxoglutarate 5-dioxygenase 1 (PLOD1 or LH1, lysyl hydroxylase 1), which leads to an abnormal pattern of urinary pyridinoline crosslinks and thus to an increased ratio of lysyl-pyridinoline to hydroxylysyl-pyridinolines. ${ }^{2}$

Recently, individuals with a clinical phenotype almost indistinguishable from PLOD1-kEDS but with a normal ratio of total urinary lysyl-pyridinoline and hydroxylysyl-pyridinolines, and causative biallelic nonsense variants in FKBP14 (MIM 614557), have been reported as a new condition, ${ }^{4}$ expanding the increasing genetic heterogeneity of EDS conditions. The first six affected individuals were reported to have muscle hypotonia at birth, progressive scoliosis, joint hypermobility, hyperelastic skin, gross motor developmental delay, myopathy, and sensorineural hearing impairment. ${ }^{4}$ Vascular complications such as dissections and occlusions in medium-sized vessels, and tortuosity of the arterial tree have been recently described in two further patients, expanding the clinical spectrum of FKBP14-kEDS. ${ }^{5,6}$ Because of severe early-onset muscle hypotonia and delayed motor development, children with FKBP14-kEDS usually undergo extensive neuromuscular workup in the neonatal period. Light and electron microscopic studies of muscle biopsies showed mild to moderate histological abnormalities and focal myofibrillar rearrangements and Z-line anomalies, respectively, albeit without a specific recognizable pattern. ${ }^{4}$ Furthermore, muscle magnetic resonance imaging (MRI) findings are indicative of fatty degeneration of muscle tissue, which is a typical feature of myopathies and muscular dystrophies. ${ }^{7,8}$ Primary myopathies caused by mutations in collagen such as the type VI (COL6) collagenopathy Ullrich congenital muscular dystrophy (MIM 254090) may show substantial connective-tissue involvement including kyphoscoliosis, joint hypermobility, joint contractures, and abnormal scar formation; ${ }^{9-13}$ however, skin hyperextensibility is absent and motor function does not improve with age. Hence, the PLOD1-kEDS and Ullrich congenital muscular dystrophy are major differential diagnoses to FKBP14-kEDS.

FKBP14 encodes for FKBP14 (alias FKBP22; Q9NWM8) a $22 \mathrm{kDa}$ protein that belongs to the FK506-binding protein (FKBP)-family of peptidyl-prolyl cis-trans isomerases (PPIases). The protein catalyzes the folding of type III collagen and interacts with type III, type VI, and type X collagens, thus helping to explain the broad phenotype observed in the patients characterized so far. ${ }^{14} \mathrm{Up}$ to now only nine patients have been reported with this ultrarare connective tissue disorder. Here we report on the clinical, biochemical, and molecular findings of an additional 17 individuals from 15 unrelated families diagnosed with FKBP14-kEDS. These individuals currently represent the largest cohort of FKBP14-kEDS and thus allow an in-depth characterization of the clinical features also including the natural history. In comparison to the clinical features reported so far, the characteristic features are emphasized and the clinical presentation is refined. The molecular data include two newly identified nonsense variants, and the hitherto first FKBP14 p.Met48Lys missense variant. The pathogenicity of this mutation is supported by conservation analysis, prediction software, and its mapping on the available crystal structure of the protein. This finding suggests that complete or partial loss of function of FKBP14 is the crucial pathological mechanism of this disorder.

\section{MATERIALS AND METHODS}

\section{Clinical and neuromuscular investigations}

Clinical information was obtained through a questionnaire that was sent to each involved center and subsequently analyzed centrally. Clinical examinations were in most cases performed by geneticists, neurologists, or pediatricians. In several cases an initially suspected diagnosis of a neuromuscular disorder could be excluded by neuromuscular workup, while in others, the differential diagnosis of PLOD1-kEDS could be ruled out by urinary pyridinoline analysis. For all studies, informed consent of the patients or their parents, in accordance with requirements of the local ethics committees of the referring physicians, was obtained.

\section{Biochemical and laboratory analyses}

Total urinary pyridinoline crosslinks, lysyl pyridinoline and hydroxylysyl pyridinoline, were measured by highperformance liquid chromatography as described. ${ }^{15,16}$

\section{Molecular genetic studies}

Genomic DNA was isolated from peripheral blood leukocytes using standard procedures.

For 14 of 17 patients, mutation analysis of the coding region and the flanking intron-exon boundaries of FKBP14 (RefSeq accession number NG_032173.1 (genomic) and NM_017946.3 (mRNA)) was performed by polymerase chain reaction and direct Sanger sequencing as previously described. ${ }^{4}$

For transcript analysis, total RNA was extracted from fibroblasts of P5/FIV and cDNA synthesis and polymerase chain reaction were performed according to standard protocols.

Targeted exome sequencing using SureSelectXT (Agilent Technologies, Santa Clara, CA, USA) and MiSeq platform (Illumina, San Diego, CA, USA) was used to analyze the coding regions and intron/exon boundaries of a panel of genes associated with kyphoscoliosis (B4GALT7, CHST14, FKBP14, PLOD1, PRDM5, RIN2, SLC39A13, and ZNF469). Mutations were confirmed by Sanger sequencing. Nucleotide numbering starts with +1 , which corresponds to the first 
ATG translation initiation codon at position 294 of the reference sequence NM_017946.3, whereas amino acid residues are numbered from the first methionine residue of the reference sequence NP_060416.1

\section{Mapping of the FKBP14 Met48Lys missense variant onto the crystal structure}

The methionine residue 48 (Met48), which was found to be mutated to lysine (Lys) in one patient, was mapped onto the 1.9- $\AA$ resolution crystal structure of human peptidyl-prolyl cis-trans isomerase FKBP14 (Protein Data Bank, 4MSP) ${ }^{17}$ using MolSoft MolBrowser 3.8-5.

\section{RESULTS}

Clinical and genetic findings of selected patients based either on newly recognized clinical features or new pathogenic variants

The disease-causing variants identified in the patient cohort are mapped onto the FKBP14 protein structure and shown in Supplementary Figure S4A online. Clinical and genetic findings of selected patients are listed in Table $\mathbf{1 .}$

\section{Patient P2/FII}

Patient 2/FII (Figure 1d) is a 9-year-old girl, born to consanguineous first-cousin parents of Iranian origin, who presented at birth with severe congenital hypotonia. She showed bilateral clubfoot; motor developmental delay; severe muscle weakness with a positive Gowers' sign; progressive severe scoliosis that required surgical intervention; severe joint hypermobility of fingers, wrists, and toes; soft skin; easy bruising; normal hearing; nasal speech; and dysarthria. Independent walking was achieved at the age of 7 years.

Sequencing of FKBP14 revealed a novel homozygous c. $143 \mathrm{~T}>\mathrm{A}$ substitution in exon 1 causing a p.(Met48Lys) mutation. Both parents were carriers of this variant. This missense mutation is not reported in the browser of the Exome Aggregation Consortium (http://exac.broadinstitute. org) and it is likely to be disease-causing according to the prediction software SIFT and Mutation Taster, or potentially benign according to PolyPhen2. The amino acid residue Met48 is conserved from human to birds (BLASTP search). Mapping of Met 48 on the three-dimensional structure of FKBP14 (PDB 4MSP) reveals that the amino acid is localized near the putative PPIase active site amino acids tyrosine 52 (Y52), isoleucine 75 (I75), tryptophan 88 (W88), and phenylalanine 127 (F127) (Supplementary Figure S4B).

\section{Patients P5/FIV and P6/FIV}

P5/FIV is the first child of healthy consanguineous firstcousin parents of Pakistani origin. He was born by emergency Cesarean section for fetal bradycardia, following spontaneous rupture of membranes and premature labor at 31 weeks gestation. On clinical examination he was noted to have a very wide cleft palate, mild micrognathia, and a large anterior fontanel. He had clenched hands with bilateral single palmar creases, overlapping fingers, and tightly adducted thumbs; mild flexion contractures of the elbows and knees; and bilateral talipes equinovarus, with prominent heels and leftsided developmental dysplasia and dislocation of the hip. $\mathrm{He}$ also had a large umbilical hernia and right inguinal hernia. $\mathrm{He}$ was breathing spontaneously at birth, but at the age of 3 days, he required intubation and mechanical ventilation. A tracheostomy was inserted because of upper airway obstruction and he was ventilated for 6 months. The tracheostomy was removed at the age of 7 years. He had gastroesophageal reflux and required long-term nasogastric feeding followed by insertion of percutaneous endoscopic gastrostomy and fundoplication at the age of 2 years. He had motor delay and first sat unsupported at the age of 12 months and walked at the age of 3 years. On examination at the age of 2 years 6 months, the limb contractures had resolved, and he had marked generalized joint laxity and poor muscle bulk. His skin was soft and velvety, but not hyperextensible. There was hypertrichosis of the arms, legs, and at the base of the spine. He had long fingers. There was pectus carinatum and kyphoscoliosis. Due to bilateral sensorineural hearing loss he received a cochlear implant in the right ear at age 6 years.

P6/FIV is the 4-year-old brother of P5/FIV. Talipes and polyhydramnios were noted antenatally. He was born by emergency Cesarean section at 38 weeks gestation and required intubation and ventilation on the first day of life. He was floppy at birth and had clenched hands and overlapping fingers. He was noted to have micrognathia, but not cleft palate. He passed his neonatal hearing screen. On examination, at the age of 7 months, he had mild hypertrichosis and esotropia. There was marked, generalized joint hypermobility and poor muscle bulk. He had "crisscross" markings on his palms and soles. He also had a small mouth and deep-set eyes. He developed gastroesophageal reflux and required nasojejunal feeding and fundoplication. He had repeated hospital admissions during infancy for respiratory tract infections and has nocturnal ventilation with bilevel positive airway pressure overnight. He had surgery for severe kyphoscoliosis at the age of 2.5 years. He had motor delay and sat unaided at the age of 2 years. At the age of 4 years he can stand and walks with support, only. His most recent hearing test showed moderate conductive hearing loss and hypermobile tympanic membranes.

Sanger sequencing confirmed that both siblings are homozygous for the c.197+5_197+ 8del mutation in intron 1 of FKBP14. Both parents were confirmed to be heterozygous carriers of the mutation. Transcript analysis on total RNA extracted from cultured fibroblasts of P5/FIV revealed that the identified deletion of 4 nucleotides in intron 1 of the genomic sequence of FKBP14 leads to the insertion of 17 nucleotides in the transcript of FKBP14 in between exons 1 and 2 and to a new open reading frame from c.198 on and a p. His67* premature termination codon ( $\mathrm{CAC}>\mathrm{TAA})$.

\section{Patient P17/XV}

This 11-month-old boy presented with micrognathia and clenched hands on antenatal ultrasound. 


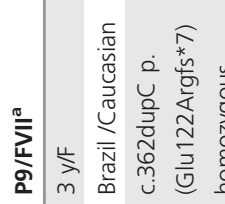

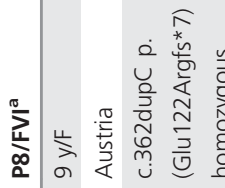

$+\frac{9}{2}+$

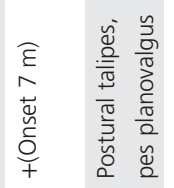

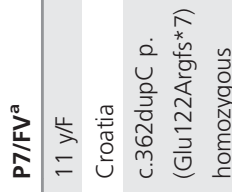

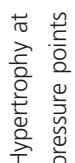

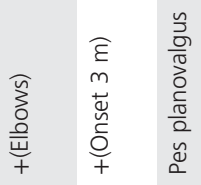

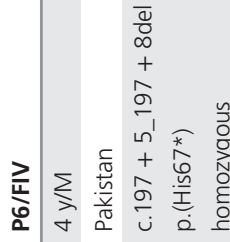

$++\frac{2}{1}$

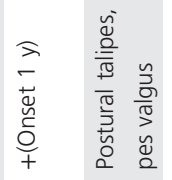

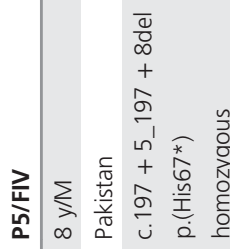

$++\frac{\infty}{z}$

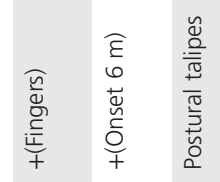

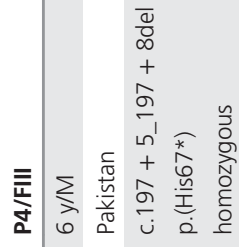

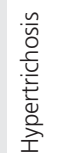

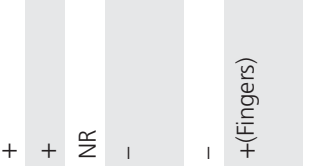

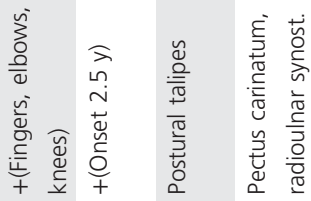

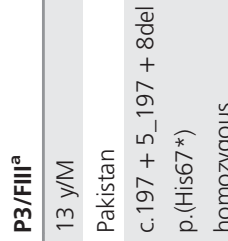

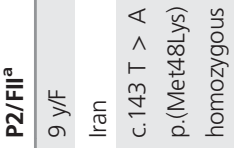

$++\stackrel{0}{\infty}+$

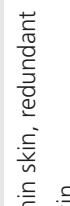

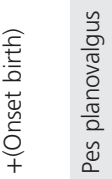

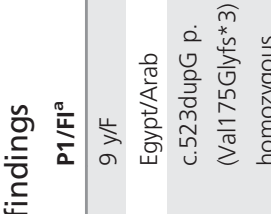

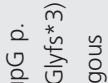
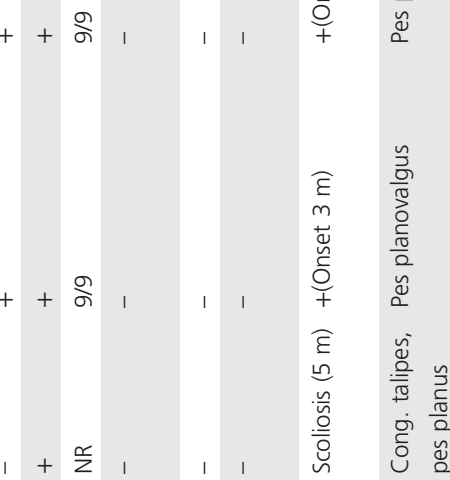

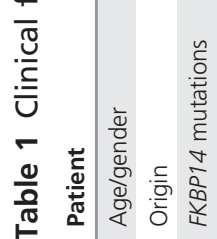
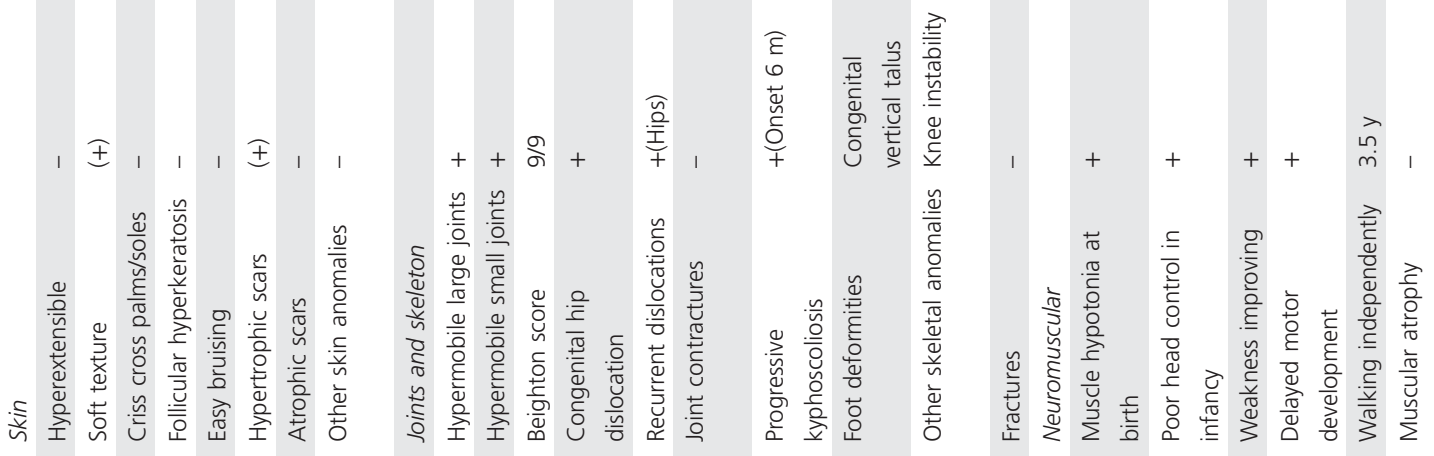


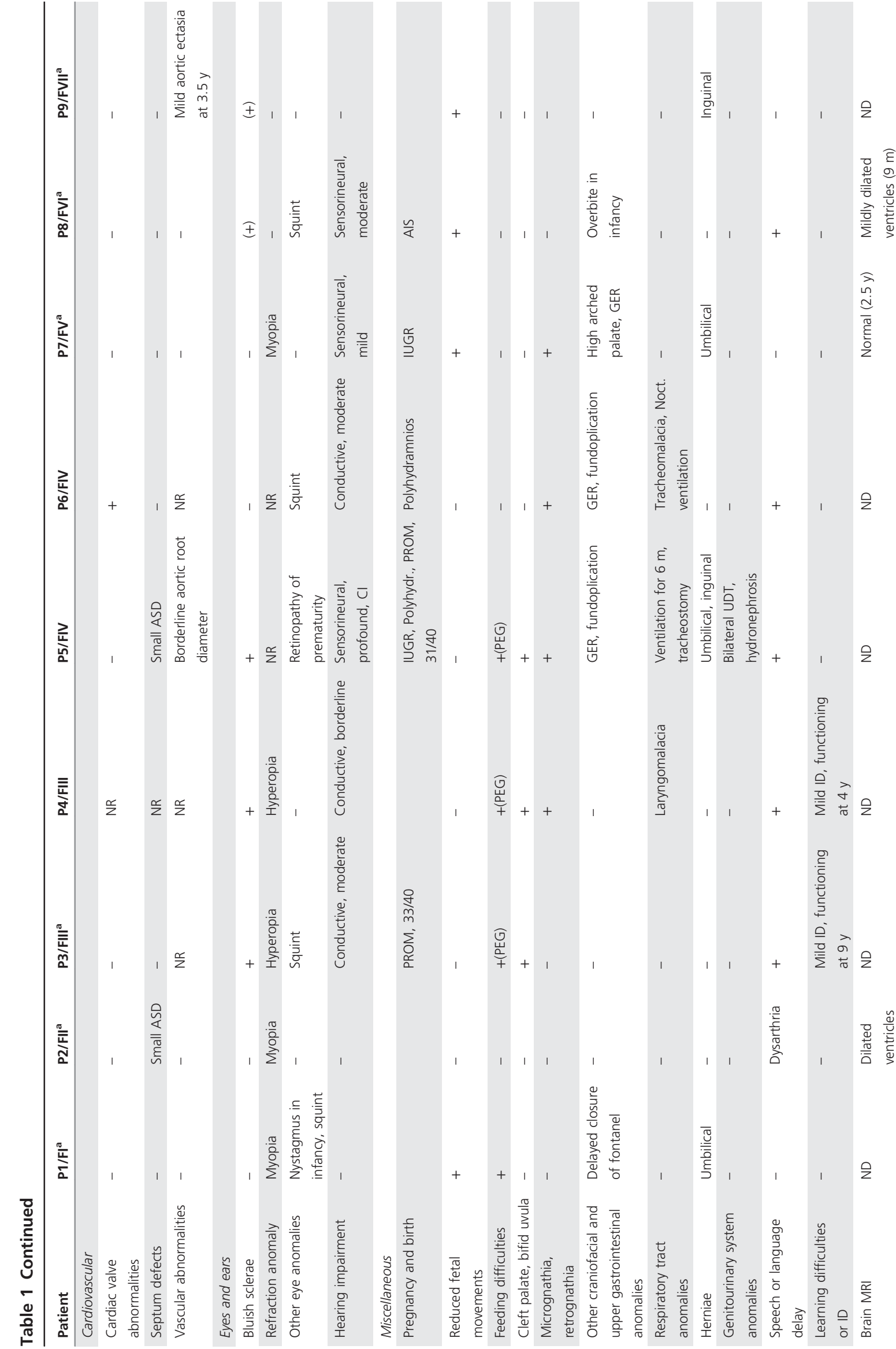



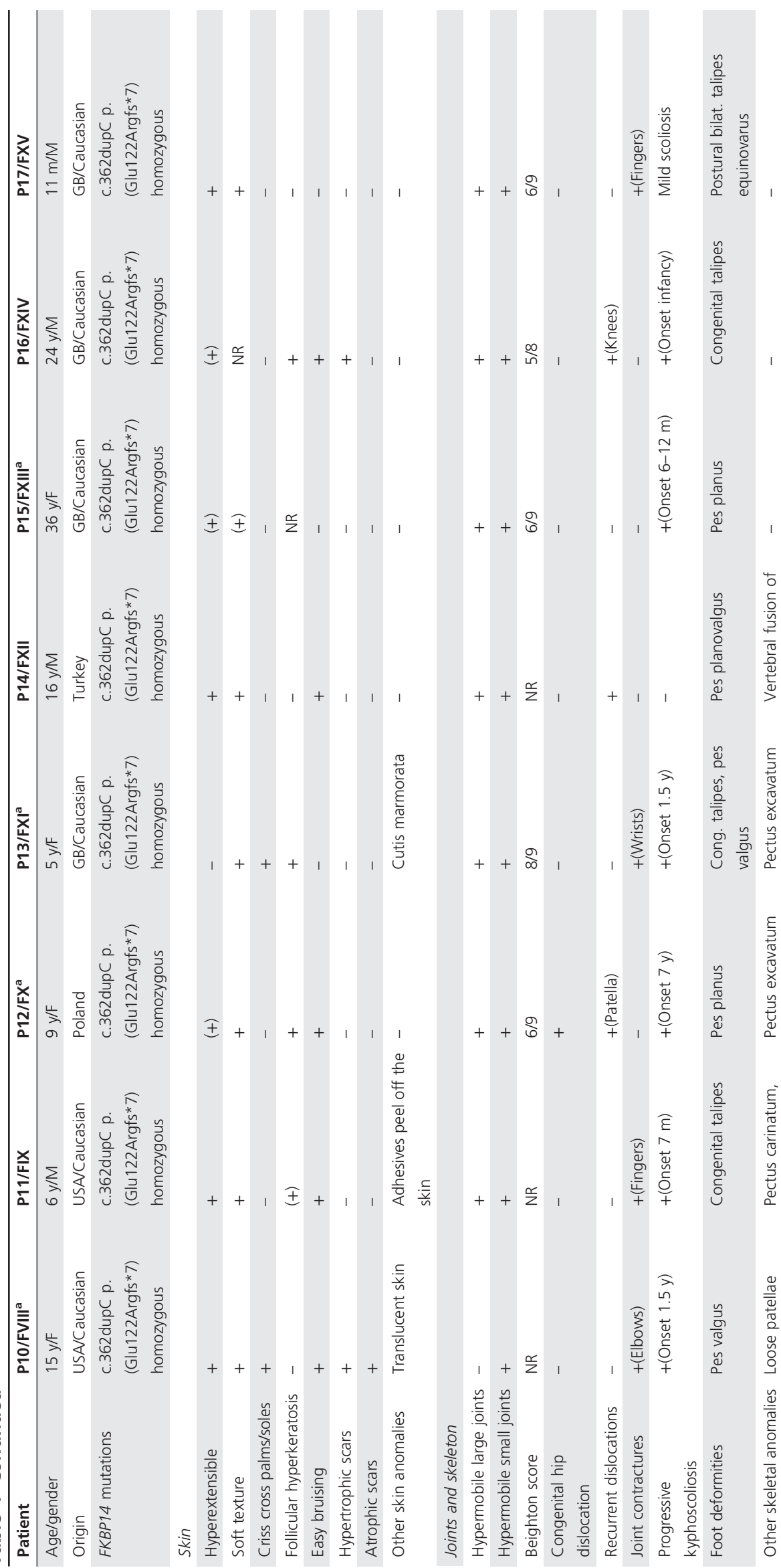

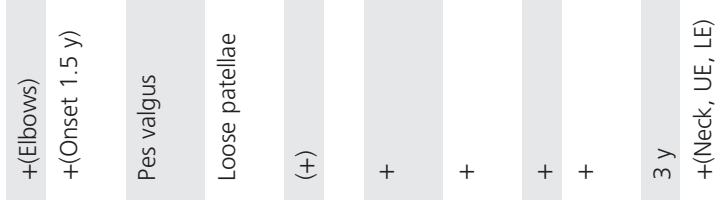

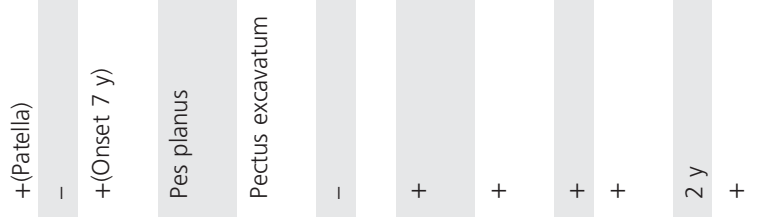



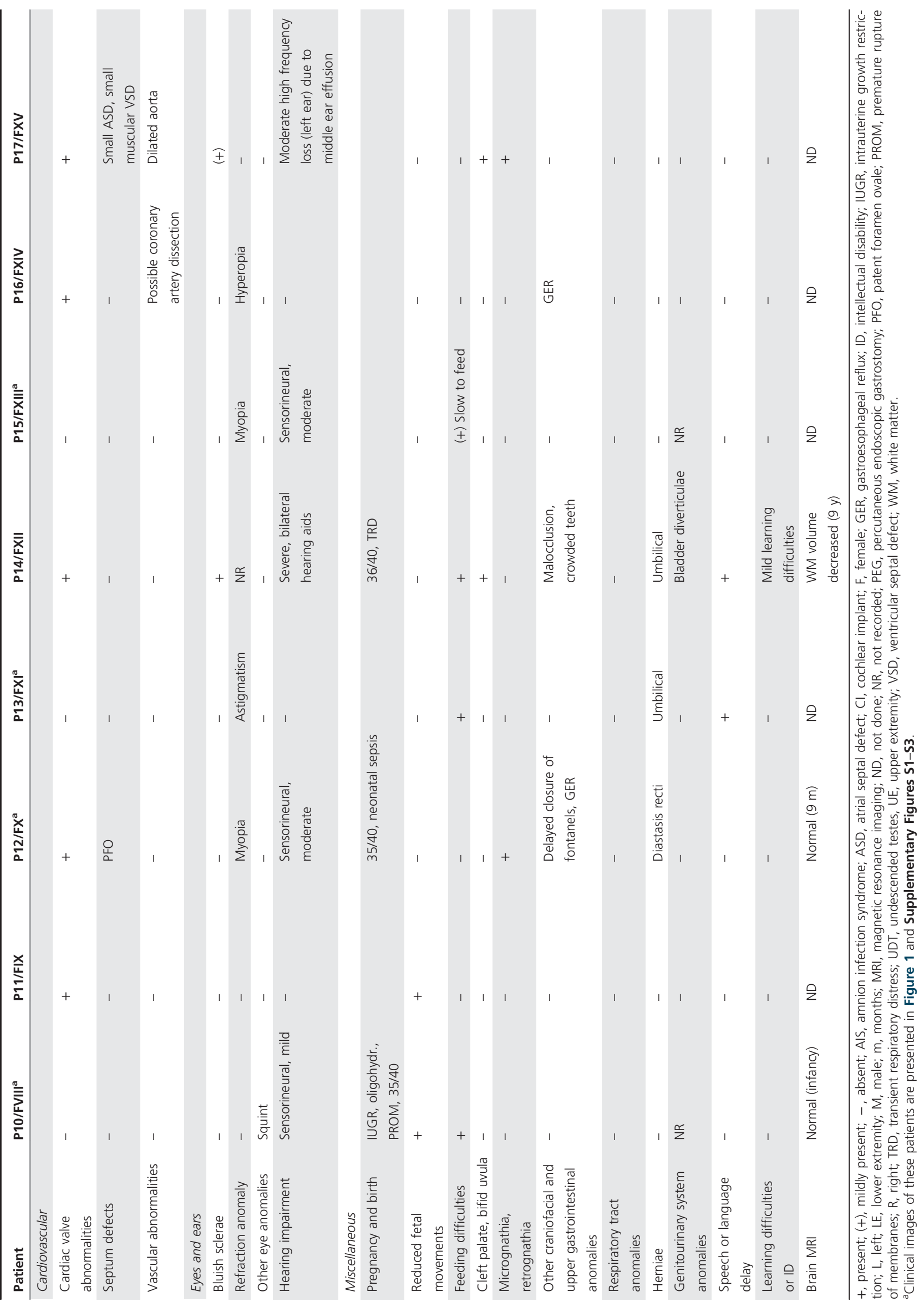

要

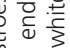

of

出

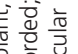

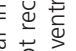

证

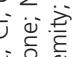

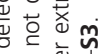

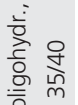

赵

䓂言

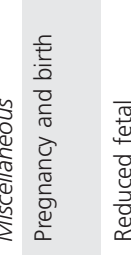

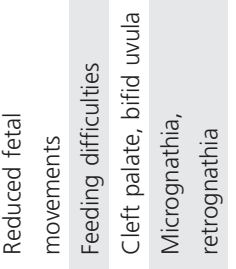

잉

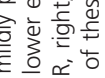

王出绖

政

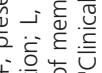




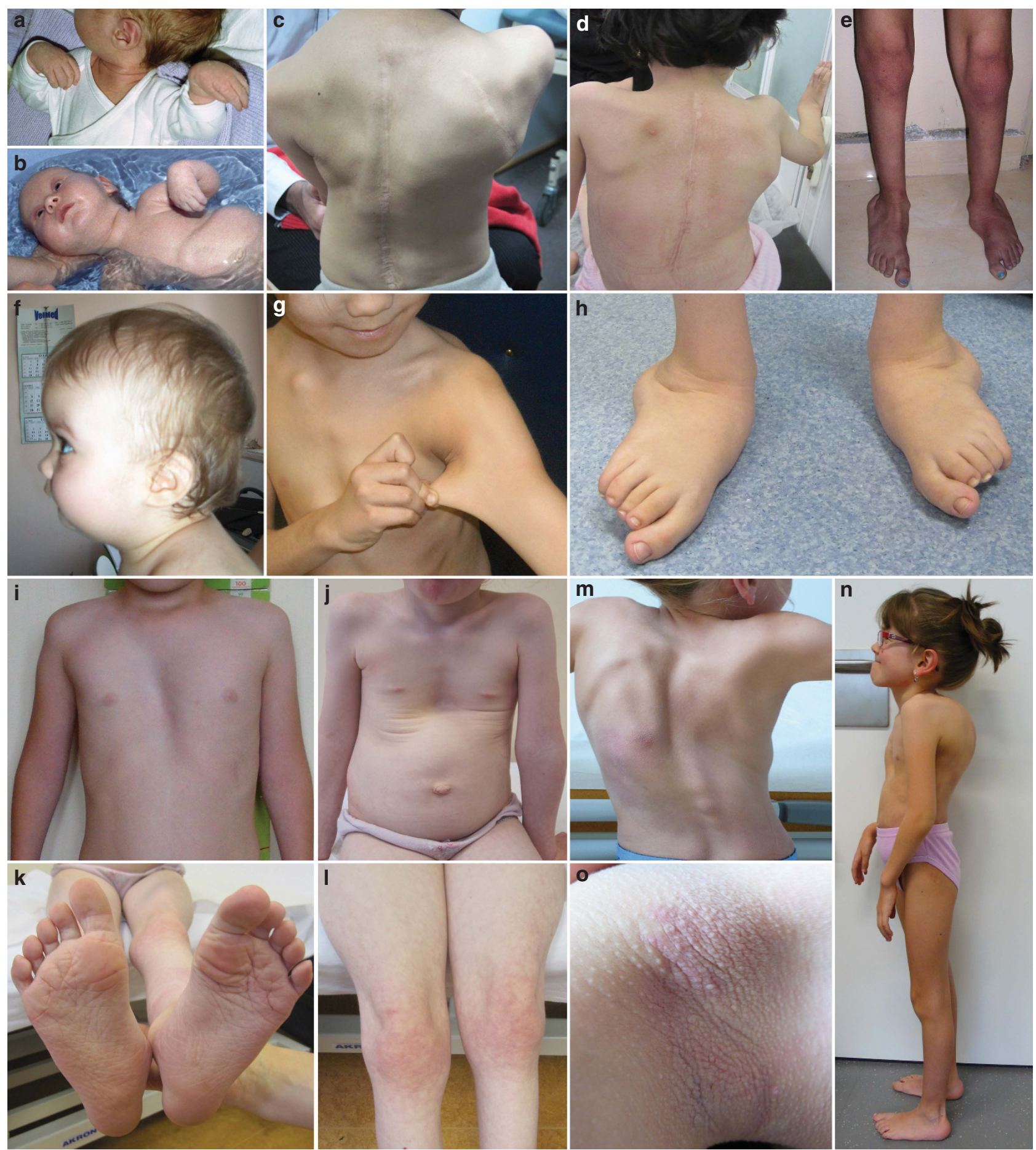

Figure 1 Clinical phenotype of patients with FKBP14-kEDS. Consent for publication of patient photos was obtained for each subject. (a,b) P1 from the original publication ${ }^{4}$ showing wrist-drop of both hands in early infancy (a,b, age 2 and 6 weeks). (c) P3/FIII at age 10 years after surgical correction of the severe thoracolumbar scoliosis. (d) P2/FIl at age 8 years after surgical correction of the progressive severe scoliosis. (e) P1/FI at age 8 years with pes planovalgus and rocker bottom feet. (f,g) P7/FV showing micrognathia (f, age 1 year), and hyperextensible skin (g, age 8 years). (h) P10/ FVIII at age 12 years with pronounced pes valgus. (i) P12/FX at age 8 years presenting with pectus excavatum. (j-I) P13/FXI at age 5 years presenting with pectus excavatum, umbilical herniae, and wrinkly, redundant skin (j), pronounced criss cross pattern of the soles ( $\mathbf{k})$ and cutis marmorata $(\mathbf{I})$. ( $\mathbf{m}-\mathbf{0})$ $\mathrm{P} 8 / \mathrm{FVI}$ at age 6 years $(\mathbf{m}, \mathbf{o})$, and 8 years $(\mathbf{n})$ showing scoliosis $(\mathbf{m})$, hyperkeratotic skin eruptions in the pressure areas under the thoracolumbar orthosis (o), and mild flexion contractures of the elbows (n). 
Postnatally he was found to have hypotonia, Pierre Robin sequence, camptodactyly of his second and third fingers bilaterally, and bilateral positional talipes equinovarus. After delivery he was transferred to the neonatal unit due to problems maintaining his saturations. He was initially managed with a nasopharyngeal airway and was fed by nasogastric tube due to concerns about his poor gag reflex. Oral feeding has subsequently been introduced. Newborn screening suggested a mild sensorineural loss on the left but more recent audiology shows normal bone conduction and a moderate hearing loss of $40 \mathrm{~dB}$ due to a middle ear effusion. An echocardiogram at a month of age showed a pseudo bicuspid valve, small muscular ventricular septal defect, normal cardiac function, and dilatation of the ascending aorta. A later echocardiogram at 10 months in addition showed a small atrial septal defect and aortic dilatation with a $Z$ score of +3.75 just beyond the tubular junction. At 9 months he was noted to have a mild thoracolumbar scoliosis to the left, which was confirmed on X-ray. On examination he was hypotonic and had just gained head control. He was not yet able to sit. The skin was hyperextensible with no evidence of scarring and no bruises. Nextgeneration sequencing for a set of selected genes (ACTA2, COL1A1, COL1A2, COL3A1, COL5A1, COL5A2, EFEMP2, FBN1, FBN2, FKBP14, FLNA, HNRNPK, MED12, MYH11, MYLK, NOTCH1, PRKG1, SKI, SLC2A10, SMAD3, SMAD4, TGFB2, TGFB3, TGFBR1 and TGFBR2) identified the common mutation c.362dupC p.(Glu122Argfs ${ }^{\star} 7$ ) in FKBP14. The parents were heterozygous for the identified variant.

\section{Biochemical, neuromuscular, and laboratory investigations (Supplementary Table S1)}

The results of laboratory, biochemical, and neuromuscular analyses are summarized in Supplementary Table S1.

\section{Follow-up and additional findings in three previously published patients (Supplementary Table S2)}

The previously reported clinical features of patients P1, P2, and $\mathrm{P} 6,{ }^{4}$ along with additional findings at follow-up, are summarized in Supplementary Table S2. At the age of 50 years $\mathrm{P} 2$ of the original publication developed an extracranial dissection of the right internal carotid artery (Figure 2a-c). The event occurred during a respiratory infection with intense coughing. The lesion was located just above the carotid artery bifurcation with formation of a pseudoaneurysm extending to the base of the skull. No cerebral infarction occurred and treatment was nonsurgical. Investigations further revealed elongation and increased tortuosity of the extracranial cervical vessels, dilatation of the ascending aorta, and mild mitral and pulmonary valve insufficiency. Echocardiography showed normal systolic and diastolic ventricular function and no signs of cardiomyopathy. Repeat MRI angiography after 9 months showed no residual pseudoaneurysm of the carotid artery but a small pseudoaneurysm of the right vertebral artery.

Because of progressive scoliosis, patient P6 underwent a spinal MRI at age 4 years, which revealed an atlantoaxial subluxation with dens dislocation and myelocompression (Figure 2d,e). Consecutively, the craniocervical junction was surgically stabilized with dorsal instrumentation. Neurological complications did not occur and at last followup at the age of 9 years she was in a stable neurological condition.

At age 16 years, patient P1 presented with exacerbation of the kyphoscoliosis with severe thoracic deformity resulting in restrictive lung disease. A dorsal thoracolumbar distraction spondylodesis was performed at the age of 17 years after preoperative treatment with halo and sling traction. Subsequent to surgery a height gain of $9 \mathrm{~cm}$ was documented, and a considerable improvement of pulmonary function (increase in forced vital capacity from 47 to $66 \%$ ) and endurance was achieved.

Echocardiography performed at the age of 19 years showed diameters of the aortic root and the ascending aorta at the upper normal limit ( $Z$ score 1.8 ) without irregularities of the wall. Echocardiography control and cardiac MRI at the age of 21 years showed a slight increase of the diameters, now above the upper normal limit ( $Z$ score 2.2).

\section{DISCUSSION}

FKBP14-kyphoscoliotic EDS (FKBP14-kEDS), caused by biallelic mutations in FKBP14, represents one of the differential diagnoses of PLOD1-kyphoscoliotic EDS (PLOD1kEDS, EDS VIA). In the first description six genetically proven and one likely affected individual were reported with this ultrarare connective-tissue disease. ${ }^{4}$ Meanwhile, three additional patients have been delineated, ${ }^{5,6,18}$ showing that the phenotypic spectrum may be broader than initially anticipated in the original publication, and raising the risk for a lifethreatening cardiovascular phenotype. Based on our results, as summarized in Table 2, the major diagnostic criteria are (i) severe generalized hypotonia at birth with marked muscle weakness that improves in infancy, and delayed gross motor milestones; (ii) early-onset progressive (kypho)scoliosis; (iii) joint hypermobility without pronounced contractures; (iv) foot deformities; and (v) normal or decreased ratio of lysyl pyridinoline to hydroxylysyl pyridinoline in urine. Minor criteria include (i) hyperelastic skin with follicular hyperkeratosis, easy bruising, and occasional abnormal scarring; (ii) myopathy as seen clinically by reduced strength and endurance and confirmed in some patients by histology and muscle imaging; and (iii) hearing impairment that is predominantly sensorineural and may not be present in all individuals. This information was seminal to the redraft of the 2017 International Nosology of EDS. ${ }^{3}$

Occasional features underlying systemic connective-tissue involvement are aortic rupture and arterial dissection, subdural hygroma (potentially due to subdural bleeding or spontaneous intracranial hypotension), ${ }^{19}$ insufficiency of cardiac valves, bluish sclerae, bladder diverticula, inguinal or umbilical herniae, and premature rupture of membranes.

We now add the clinical, biochemical, and molecular description of 17 additional patients, and describe new clinical 

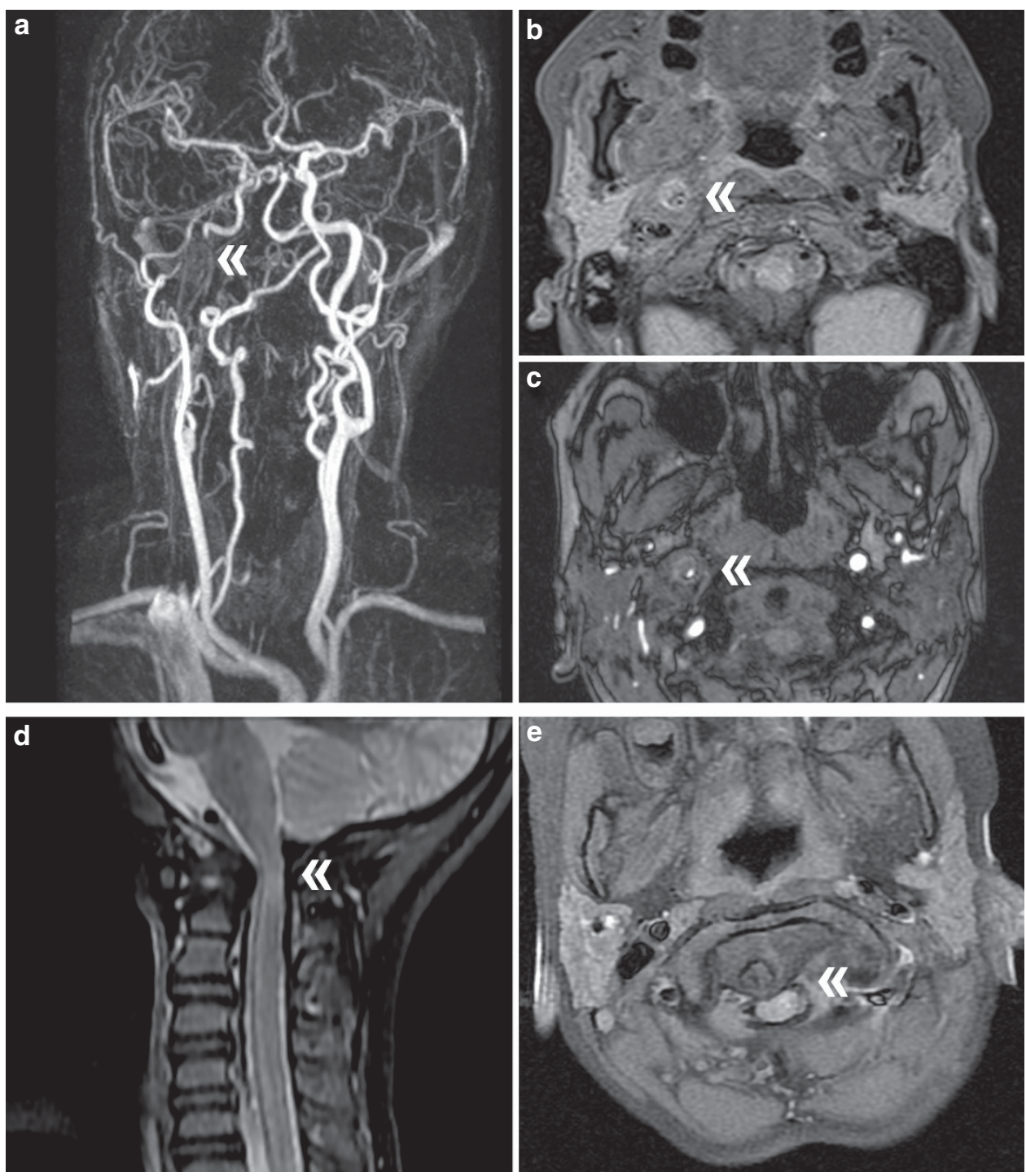

Figure 2 Radiological findings. At the age of 50 years, P2 from Baumann et al. ${ }^{4}$ developed a dissection of the internal carotid artery. The perforation was located just above the right carotid artery bifurcation with formation of a pseudoaneurysm extending to the base of the skull. (a) Contrastenhanced magnetic resonance angiography shows interruption of the right internal carotid artery (double arrowhead) and elongation and increased tortuosity of the extracranial vessels. Axial sections with (b) fat saturated T1 sequences and (c) time-of-flight angiography show the residual lumen of the right internal carotid artery (double arrowhead). Spinal magnetic resonance image at age 4 years in P6 from Baumann et al. ${ }^{4}$ shows an atlantoaxial subluxation with dens dislocation and myelocompression. (d) In sagittal T2-weighted imaging compression of the spinal canal and increased signal intensity of the myelon at the craniocervical junction as sign of myelopathy (double arrowhead). (e) Axial T1-weighted imaging shows dislocation of the dens with myelocompression (double arrowhead) and pannus proliferation around the dens.

features uncovered in three patients, originally published in 2012. ${ }^{4}$ This observational data emphasizes the clinical features, allowing for the definition of major and minor diagnostic criteria and adds insight into the clinical variability of the disease. It is certainly of note that the molecular diagnosis in the majority of the patients was achieved by direct Sanger sequencing of FKBP14 due to the strong suggestive clinical features.

The risk of vascular complications in FKBP14-kEDS was initially suspected based on the history of aortic rupture in the 12-year-old sibling of a proven affected patient (P2). ${ }^{4}$ She had shared characteristic clinical features with her similarly affected sister but had died prior to molecular testing. The follow-up in her sister at the age of 50 years revealed an internal carotid artery dissection. This finding strongly supports the suspicion of a vascular phenotype of FKBP14kEDS; vascular complications including a dissection of the celiac artery at age 41 years $^{6}$ and a hypogastric artery pseudoaneurysm rupture at age 6 years ${ }^{5}$ were described recently. Therefore awareness for possible serious vascular complications in different age groups in patients with FKBP14-kEDS should be raised, and vascular screening already starting at pediatric age should be included into the surveillance of these patients. 
Clinical follow-up of the initial cohort ${ }^{4}$ revealed a new skeletal feature in one patient. Symptomatic atlantoaxial subluxation with dens dislocation and myelocompression requiring a posterior occipitocervical fixation was found in P6 at age 4 years. Asymptomatic atlantoaxial instability was recently reported in a 6-year-old patient, ${ }^{5}$ but not in any other patient known so far, although not all had radiological investigations aimed to investigate this feature. Occipitoatlantoaxial hypermobility and instability has been described in other types of EDS, in particular in the classic, hypermobile, and vascular type. ${ }^{20-23}$

In the vast majority of the patients of this cohort the hands have a normal appearance (Supplementary Figure S1).

Limb contractures were not observed in the initial patient group $^{4}$ and the three single patients delineated subsequently. ${ }^{5,6,18}$ However, the cohort presented here includes seven patients with prominent congenital contractures of fingers, wrist, elbows, or knees. Although the natural history of the contractures cannot be predicted yet, amelioration was documented in half of the affected, whereas two remained symptomatic at the age of 9 and 15 years, respectively. More pronounced contractures are a feature of the musculocontractural type of $\mathrm{EDS}^{24}$ and the collagen VI-related myopathies in which they are often progressive., ${ }^{9}$ Furthermore, extension deficit of the elbow/congenital flexion contractures are nosology criteria for the Marfan. ${ }^{25}$

Midline defects of the palate or lips are only sporadically described in EDS. ${ }^{26-28}$ However, bifid uvula and cleft palate, which are frequently found in Loeys-Dietz syndrome, ${ }^{29}$ have been observed in FKBP14-kEDS patients. A cleft of the soft palate was observed in two patients of the first patient group, and a bifid uvula in the patient described by Dordoni et al. ${ }^{5}$ In the actual patient cohort, cleft palate, defined as bifid uvula with submucous cleft palate or wide cleft palate, was found in five patients. Therefore, these observations raise the question of whether Marfan spectrum disorders, in particular Loeys-Dietz syndrome, should be considered in the differential diagnosis of FKBP14-kEDS. However, cleft palate might also occur secondary to micrognathia and retrogenia as described in three of the five patients with cleft palate in the actual cohort (P4/FIII, P5/FIV, and P17/ FIV). Hearing impairment, in particular sensorineural hearing loss, was initially described as a characteristic clinical feature of the disorder based on the findings of the initial cohort. ${ }^{4}$ Extended descriptions of additional patients indicate that this feature might be less prominent as initially suggested, as $41 \%$ currently present with sensorineural hearing impairment. However, in $18 \%$ of the patients of the present cohort, conductive hearing loss was diagnosed. Therefore, hearing impairment, although less common than previously considered, still represents a diagnostic feature of FKBP14-kEDS. This can manifest at birth or in early infancy, or even later in life. We should point out that the mainly younger age of the patients in this cohort might represent a limitation of our study in regard to the frequency of hearing impairment.
The majority of the patients in this cohort presented with congenital hypotonia and weakness, which improved during childhood. All had delayed motor developmental and reduced muscle strength, and the age of unassisted walking was delayed in all, ranging from 2 to 7 years. Nerve conduction measured in 10 patients was normal in all. Unspecific myopathic changes were found on muscle biopsies and/or electromyography in five patients, whereas muscle biopsy and/or electromyography in six patients were normal. In one patient a repeated electromyography showed an improvement of the myopathic features, and in another patient who presented repeatedly with myopathic alterations at ages 3 months and 1 year, a third electromyography was normal at age 8 years. Creatine kinase was normal and thus not to be used as a discriminating biomarker, from Bethlem myopathy and Ullrich myopathy. However, Bethlem myopathy and Ullrich congenital muscular dystrophy have distinct patterns of muscle involvement on MRI, which have not been seen in FKBP14-kEDS. Muscle MRI was performed in three patients and revealed mild atrophy of the rectus femoris, and mild hypertrophy of the vastus medialis in one of them. The clinical symptoms suggesting a myopathy are most likely caused by secondary effects of the connective alterations in and around muscle, with secondary effects on muscle fibers themselves, as is in fact the case in the COL6-related dystrophies, ${ }^{30}$ and thus not due to a primary myofiber involvement. However, in contrast to the COL6-related dystrophies, in FKBP14-kEDS this matrix abnormality does not appear to result in progressive muscle degeneration. Similar neuromuscular findings are also seen in other EDS types in particular in PLOD1-kEDS (EDS VIA). The natural course of the neuromuscular phenotype is yet to be defined. Reduced motor function and fatigue in adulthood was only reported in two adult patients ${ }^{4,6}$ and might represent an age-dependent feature in the natural course of FKBP14-kEDS.

Mild intellectual disability and/or learning difficulties have been documented in the siblings of family III and in patient P14/XII. However, in absence of additional data, we should consider that impaired cognitive development in these patients might have a different genetic cause due to parental consanguinity.

The management of patients affected with FKBP14-kEDS, with focus on the musculoskeletal, the cardiovascular, and the hearing systems, and the molecular genetic confirmation of the diagnosis, should follow the guidelines of the 2017 International Nosology of EDS. ${ }^{3}$

Ishikawa and Bächinger ${ }^{14}$ recently published evidence supporting the involvement of FKBP14, alias FKBP22, in the molecular ensemble for type III, type VI, and type X collagen maturation, showing that the protein interacts with these types of collagens. Remarkably, mutations in type III and type VI collagens are the genetic hallmark of the vascular type of EDS (vEDS) and of COL6-related dystrophies, respectively. Therefore, the clinical features of vascular abnormalities and myopathy documented in the affected individuals clearly correlates with the interaction of FKBP14 with type III and VI collagens. 
Table 2 Features associated with FKBP14 mutations in our two patient cohorts (frequency reported as percentage)

Cohorts

Total number of patients

Skin

Hyperextensible

Soft texture

Criss cross palms/soles

Follicular hyperkeratosis

Easy bruising

Hypertrophic scars

Atrophic scars

Joints and skeleton

Hypermobile large joints

Hypermobile small joints

Congenital hip dislocation

Recurrent dislocations

Joint contractures

Progressive kyphoscoliosis

Foot deformities

Fractures

Neuromuscular

Muscle hypotonia at birth

Poor head control in infancy

Weakness improving

Delayed motor development

Muscular atrophy

Cardiovascular

Cardiac valve abnormalities

Septum defects

Vascular abnormalities

Eyes and ears

Bluish sclerae

Refraction anomaly

Hearing impairment

Miscellaneous

Cleft palate, bifid uvula

Micrognathia, retrognathia

Herniae

Bladder diverticulum

Speech or language delay

Learning difficulties/intellectual disability

NR, not recorded.

ancluding follow-up in three patients.

Complete or partial loss of function of FKBP14 as the crucial disease mechanism of the disorder is suggested by mapping of the first identified missense variant p.(Met48Lys) onto the protein crystal structure (Supplementary Figure S4B). The p.Met48Lys variant maps near the potential PPIase active site of the FKBP domain. Replacement of the hydrophobic methionine by the positively charged lysine may selectively impair the PPIase activity without affecting chaperone function.
Present study

$12 / 17$
$16 / 16$
$5 / 17$
$6 / 16$
$7 / 16$
$3 / 17$
$2 / 17$

$15 / 17$

$17 / 17$

$4 / 17$

$4 / 17$

$14 / 17$

$17 / 17$

$2 / 17$

$17 / 17$

$17 / 17$

$16 / 16$

$17 / 17$

$8 / 16$

$6 / 16$

$3 / 16$

$4 / 14$

$7 / 17$

$9 / 14$

$11 / 17$

$5 / 17$

$6 / 17$

$1 / 15$

$7 / 17$

$3 / 17$
Baumann et al. ${ }^{4}$ a

6

$\begin{array}{rrr}5 / 6 & 17 / 23 & 73 \\ 6 / 6 & 23 / 23 & 100 \\ N R & 5 / 17 & 29 \\ 4 / 6 & 10 / 23 & 43 \\ 3 / 6 & 10 / 23 & 43 \\ 1 / 6 & 4 / 23 & 17 \\ 1 / 6 & 3 / 23 & 13\end{array}$

$6 / 6$

$6 / 6$

NR

$1 / 6$

$0 / 6$

$4 / 6$

$6 / 6$

$1 / 6$

$6 / 6$

$6 / 6$

$6 / 6$

$6 / 6$

$6 / 6$

$3 / 6$

$0 / 6$

$2 / 6$

$1 / 6$

$3 / 6$

$6 / 6$

$2 / 6$

$2 / 6$

$4 / 6$

$2 / 4$

$0 / 3$

$1 / 6$
Total

23

$3 / 23$

$21 / 23 \quad 91$

$23 / 23 \quad 100$

$4 / 17 \quad 23$

$5 / 23 \quad 21$

$7 / 23 \quad 30$

$18 / 23$

$23 / 23$

$3 / 23$

$23 / 23 \quad 100$

$23 / 23 \quad 100$

$22 / 22$

$23 / 23$

$14 / 22$

$9 / 22$

$3 / 22$

$6 / 20$

$8 / 23$

$12 / 20$

$17 / 23$

$7 / 23$

$8 / 23$

$11 / 23$

$3 / 19$

$7 / 20$

$4 / 23$
91

23

21
30

78

100

13

00
00

100
100

63

40

13

13

30

34

60

60

73

30

34

47

15

35

17
In summary, the review of the present patient cohort, the follow-up of three patients from our initial publication as well as the comparison with all published cases, reveals a quite consistent clinical presentation of the disorder and allows us to speculate on major and minor clinical criteria. In contrast to the broader clinical spectrum of phenotypic presentation in PLOD1-kEDS, FKBP14-kEDS thus far presents with a less pronounced phenotypical variability. Vascular complications in adulthood should be considered as a disease-related feature, 
and their possible occurrence in childhood urges cardiovascular investigations in the routine assessment and follow-up of these patients. Hearing impairment, either conductive or sensorineural, might manifest later in life or remain subclinical, thus requiring periodic investigations. Motor developmental delay and reduced motor strength are common neuromuscular features in childhood, and a decline of motor function in adulthood seems to represent an age-dependent feature in the natural course of FKBP14-deficient EDS.

\section{SUPPLEMENTARY MATERIAL}

Supplementary material is linked to the online version of the paper at http://www.nature.com/gim

\section{ACKNOWLEDGMENTS}

This work has been supported by the Swiss National Science Foundation grant no. 310030_138288 to C.G. and M.R., and for clinical assessment of patients from institutional grant KNW-1086/K/4/0 to J.P., and by intramural funds of the National Institute for Neurological Disorders and Stroke/National Institutes of Health to C.G.B. We express our gratitude to all families who participated in this study. We thank Christine Plüss and Angelika Schwarze for skillful technical assistance.

\section{DISCLOSURE}

The authors declare no conflict of interest.

\section{REFERENCES}

1. Beighton P, De Paepe A, Steinmann B, Tsipouras P, Wenstrup, R.J. EhlersDanlos syndromes: revised nosology, Villefranche, 1997. Am J Med Genet 1998;77:31-37.

2. Steinmann $B$, Royce PM, Superti-Furga $A$. The Ehlers-Danlos syndrome. In Royce PM and Steinmann B (eds). Connective Tissue and Its Heritable Disorders, 2nd edn, Wiley-Liss: New York, 2002:431-523.

3. Malfait $F$, Belmont $B$, Berglund $B$, et al. The 2017 international classification of the Ehlers-Danlos syndromes. Am J Med Genet C 2017;175: 70-115.

4. Baumann M, Giunta C, Krabichler B, et al. Mutations in FKBP14 cause a variant of Ehlers-Danlos syndrome with progressive kyphoscoliosis, myopathy, and hearing loss. Am J Hum Genet 2012;90:201-216.

5. Dordoni C, Ciaccio C, Venturini M, Calzavara-Pinton P, Ritelli M, Colombi M. Further delineation of FKBP14-related Ehlers-Danlos syndrome: a patient with early vascular complications and non-progressive kyphoscoliosis, and literature review. Am J Med Genet A 2016:170:2031-2038.

6. Murray ML, Yang M, Fauth C, Byers PH. FKBP14-related Ehlers-Danlos syndrome: expansion of the phenotype to include vascular complications. Am J Med Genet A 2014;164:1750-1755.

7. Mercuri $E$, Jungbluth $H$, Muntoni F. Muscle imaging in clinical practice: diagnostic value of muscle magnetic resonance imaging in inherited neuromuscular disorders. Curr Opin Neurol 2005;18:526-537.

8. Wattjes MP, Kley, RA, Fischer D. Neuromuscular imaging in inherited muscle diseases. Eur Radiol 2010;20:2447-2460.

9. Bönnemann CG. The collagen VI-related myopathies: muscle meets its matrix. Nat Rev Neurol 2011;21:379-390.

10. Kirschner J, Hausser I, Zou Y, et al. Ullrich congenital muscular dystrophy: connective tissue abnormalities in the skin support overlap with EhlersDanlos syndromes. Am J Med Genet A 2005;132:296-301.

11. Lampe AK, Bushby KM. Collagen VI related muscle disorders. J Med Genet 2005;42:673-685.

12. Nadeau A, Kinali M, Main M, et al. Natural history of Ullrich congenital muscular dystrophy. Neurology 2009;73:25-31.
13. Voermans NC, Bönnemann CG, Huijing PA, et al. Clinical and molecular overlap between myopathies and inherited connective tissue diseases. Neuromuscul Disord 2008;18:843-856.

14. Ishikawa $Y$, Bächinger HP. A substrate preference for the rough endoplasmic reticulum resident protein FKBP22 during collagen biosynthesis. J Biol Chem 2014;289:18189-18201.

15. Giunta C, Randolph A, Al-Gazali L, Brunner HG, Kraenzlin ME, Steinmann, B. Nevo syndrome is allelic to the kyphoscoliotic type of the Ehlers-Danlos syndrome (EDS VIA). Am J Med Genet 2005;133:158-164.

16. Kraenzlin ME, Kraenzlin CA, Meier C, Giunta C, Steinmann B. Automated HPLC assay for urinary collagen cross-links: effect of age, menopause, and metabolic bone diseases. Clin Chem 2008:54:1546-1553.

17. Boudko SP, Ishikawa Y, Nix J, Chapman MS, Bachinger HP. Structure of human peptidyl-prolyl cis-trans isomerase FKBP22 containing two EF-hand motifs. Protein Sci 2014;23: 67-75.

18. Aldeeri AA, Alazami AM, Hijazi H, Alzahrani F, Alkuraya FS. Excessively redundant umbilical skin as a potential early clinical feature of Morquio syndrome and FKBP14-related Ehlers-Danlos syndrome. Clin Genet 2014:86:469-472.

19. Schievink WI, Gordon OK, Tourje, J. Connective tissue disorders with spontaneous spinal cerebrospinal fluid leaks and intracranial hypotension: a prospective study. Neurosurgery 2004;54:65-70.

20. Halko GJ, Cobb R, Abeles M. Patients with type IV Ehlers-Danlos syndrome may be predisposed to atlantoaxial subluxation. I Rheumatol 1995;22:2152-2155.

21. Karaa A, Stoler JM. Ehlers Danlos syndrome: an unusual presentation you need to know about. Case Rep Pediatr. 2013;2013:764659.

22. Milhorat TH, Bolognese PA, Nishikawa M, McDonnell NB, Francomano CA Syndrome of occipitoatlantoaxial hypermobility, cranial settling, and Chiari malformation type I in patients with hereditary disorders of connective tissue. J Neurosurg Spine 2007;7:601-609.

23. Nagashima C, Tsuji R, Kubota S, Tajima K. Atlanto-axial, atlanto-occipital dislocations, developmental cervical canal stenosis in the Ehlers-Danlos syndrome. No Shinkei Geka 1981;9:601-608.

24. Janecke $A R$, Li B, Boehm $M$, et al. The phenotype of the musculocontractural type of Ehlers-Danlos syndrome due to CHST14 mutations. Am J Med Genet A 2016;170:103-115.

25. von Kodolitsch $Y$, De Backer J, Schüler $\mathrm{H}$, et al. Perspectives on the revised Ghent criteria for the diagnosis of Marfan syndrome. Appl Clin Genet 2015;8:137-155.

26. Okamura $\mathrm{H}$, Matsumoto $Y$. A case of Ehlers-Danlos syndrome associated with cleft lip and palate. J Laryngol Otol 1984;98:311-315.

27. Rizzo R, Contri MB, Micali G, Quaglino D, Pavone L, Ronchetti IP. Familial Ehlers-Danlos syndrome type II: abnormal fibrillogenesis of dermal collagen. Pediatr Dermatol 1987;4:197-204.

28. Tan PW, Song C, Lalonde D. Ehlers-Danlos syndrome associated with cleft lip and palate. Can J Plast Surg 2009;17:e24-6.

29. Van Laer L, Dietz H, Loeys B. Loeys-Dietz syndrome. Adv Exp Med Biol 2014;802:95-105

30. Zou Y, Zhang RZ, Sabatelli P, Chu ML, Bönnemann CG. Muscle interstitial fibroblasts are the main source of collagen VI synthesis in skeletal muscle: implications for congenital muscular dystrophy types Ullrich and Bethlem. J Neuropathol Exp Neurol 2008;67:144-154.

\section{This work is licensed under a Creative Commons} Attribution-NonCommercial-ShareAlike $\quad 4.0$ International License. The images or other third party material in this article are included in the article's Creative Commons license, unless indicated otherwise in the credit line; if the material is not included under the Creative Commons license, users will need to obtain permission from the license holder to reproduce the material. To view a copy of this license, visit http://creativecommons.org/licenses/ by-nc-sa/4.0/

(C) The Author(s) 2018 